\title{
Cation induced differential effect on structural and functional properties of Mycobacterium tuberculosis $\alpha$-Isopropylmalate synthase
}

\author{
Kulwant Singh and Vinod Bhakuni*
}

Address: Division of Molecular and Structural Biology, Central Drug Research Institute, Lucknow-226 001, India (CDRI communication number7085)

Email: Kulwant Singh - ksinghcdri@gmail.com; Vinod Bhakuni* - bhakuniv@rediffmail.com

* Corresponding author

Published: 19 June 2007

BMC Structural Biology 2007, 7:39 doi:10.1 186/1472-6807-7-39

This article is available from: http://www.biomedcentral.com/1472-6807/7/39

(c) 2007 Singh and Bhakuni; licensee BioMed Central Ltd.

This is an Open Access article distributed under the terms of the Creative Commons Attribution License (http://creativecommons.org/licenses/by/2.0), which permits unrestricted use, distribution, and reproduction in any medium, provided the original work is properly cited.

\begin{abstract}
Background: $\alpha$-isopropylmalate synthase (MtalPMS), an enzyme that catalyzes the first committed step of the leucine biosynthetic pathway of Mycobacterium tuberculosis is a potential drug target for the anti-tuberculosis drugs. Cations induce differential effect of activation and inhibition of MtalPMS. To date no concrete mechanism for such an opposite effect of similarly charged cations on the functional activity of enzyme has been presented.

Results: Effect of cations on the structure and function of the MtalPMS has been studied in detail. The studies for the first time demonstrate that different cations interact specifically at different sites in the enzyme and modulate the enzyme structure differentially. The inhibitors $\mathrm{Zn}^{2+}$ and $\mathrm{Cd}^{2+}$ ions interact directly with the catalytic domain of the enzyme and induce unfolding/denaturation of the domain. The activator $\mathrm{K}^{+}$also interacts with the catalytic TIM barrel domain however, it does not induce any significant effect on the enzyme structure. Studies with isolated catalytic TIM barrel domain showed that it can carry out the catalytic function on its own but probably requires the non-catalytic C-terminal domain for optimum functioning. An important observation was that divalent cations induce significant interaction between the regulatory and the catalytic domain of MtalPMS thus inducing structural cooperativity in the enzyme. This divalent cation induced structural cooperativity might result in modulation of activity of the catalytic domain by regulatory domain.
\end{abstract}

Conclusion: The studies for the first time demonstrate that different cations bind at different sites in the enzyme leading to their differential effects on the structure and functional activity of the enzyme.

\section{Background}

Tuberculosis is the second leading infectious cause of mortality worldwide. Mycobacterium tuberculosis remains one of mankind's deadliest pathogen, responsible for approximately two billion deaths worldwide every-year, which is one-third of the world's population [1]. Although effective drugs against tuberculosis exist, therapy requires prolonged treatment with several drugs, leading to problems in compliance and emergence of multidrug resistance [2]. There is an urgent need for more 
effective drugs against tuberculosis. Hence, development of new drugs and characterization of new targets is urgently required.

Mycobacteria synthesize the branched-chain amino acids, L-valine, L-leucine and pantothenic acid from $\alpha$-ketoisovalerate ( $\alpha$-KIV). The essentiality of this pathway in $M$. tuberculosis, and its absence in humans makes the enzymes of this pathway attractive target/s for development of drug/s for treatment of tuberculosis [3]. The first step in the L-leucine biosynthesis is the formation of $\alpha$-isopropylmalate from acetyl-CoA and $\alpha$-KIV that is catalyzed by $\alpha$ isopropylmalate synthase ( $\alpha$-IPMS). $\alpha$-IPMS is an allosteric enzyme that is present in various organisms like bacteria, fungi and plants.

Crystal structure of only one $\alpha$-IPMS, Mt $\alpha$ IPMS a dimeric enzyme, has been reported to date [4]. Each monomer of the enzyme is folded into two major N- and C-terminal domains which are separated by two small sub-domains, sub domain I and sub domain II that are joined by a flexible hinge [4]. The N-terminal domain contains the active site and the C-terminal domain the L-leucine binding site of the enzyme. The functional characterization of $\alpha$-IPMS from several organisms have been reported [5-8]. They share some common features like requirement of monovalent cations for activity, feedback inhibition by L-leucine and narrow substrate specificity for analogues of $\alpha$ KIV. The kinetic parameters of the substrates for MtaIPMS are significantly influenced by cations both monovalent and divalent. The $\mathrm{K}^{+}$is physiological activator of the enzyme $[9,10]$. Divalent cations show broad specificity for the functional activity of the enzyme. $\mathrm{Mg}^{2+}$ and $\mathrm{Mn}^{2+}$ induce activation whereas; $\mathrm{Zn}^{2+}$ and $\mathrm{Cd}^{2+}$ induce inhibition of the functional activity of the enzyme [9]. The kinetics of activation or inhibition of the MtaIPMS by the cations has been extensively studied and possible mechanisms have been proposed. However, no experimental validations of the proposed mechanism/s have yet been documented.

In order to understand the mechanism of modulation of functional activity of Mt $\alpha$ IPMS by cations we have carried out detailed functional and structural studies. For studying the specificity of interaction of cations with different domains of the enzyme, the catalytic TIM barrel domain was isolated and purified. The effects of cations on the structural and functional properties of the isolated TIM barrel domain were carried out. Comparative analysis of the effect of cations on the isolated catalytic domain and the full-length enzyme provides intriguing insight into the possible mechanism of cation induced changes in the Mt $\alpha$ IPMS.

\section{Results \\ Over-expression and purification of Mt $\alpha$ IPMS}

The expression of the recombinant MtaIPMS was good and the expressed protein was present predominantly $(>90 \%)$ in the soluble fraction (Figure 1). The purified protein was homogenous as indicated by a single protein band on SDS-PAGE 1 (Figure 1) and a single peak in ESIMS of molecular mass about 73.1 kDa (data not shown).

\section{Differential effect of metal cations on the functional activity of Mt $\alpha$ IPMS}

Metal cations, both monovalent and divalent, showed broad specificity on the functional activity of MtaIPMS (Figure 2A). Monovalent cation $\mathrm{K}^{+}$, was essential for the functional activity of the enzyme as in its absence no activity was observed. Presence of $\mathrm{Mg}^{2+}$ ions along with $\mathrm{K}^{+}$ resulted in enhancement (about 115\%) of functional activity. $\mathrm{Zn}^{2+}$ and $\mathrm{Cd}^{2+}$ ions induced significant inhibition of above $90 \%$ of the functional activity of Mt $\alpha$ IPMS. These observations are similar to the earlier report [9].

\section{Cations induce differential effects on the secondary structure of Mt $\alpha$ IPMS}

The effect of cations on the structure of MtaIPMS was studied by monitoring changes in the far-UV CD of enzyme at $222 \mathrm{~nm}$ in presence of increasing concentration of $\mathrm{MgCl}_{2}, \mathrm{KCl}, \mathrm{ZnCl}_{2}$ and $\mathrm{CdCl}_{2}$ (Figure 3). No significant alteration in the secondary structure of the enzyme was observed in presence of $\mathrm{MgCl}_{2}$ and $\mathrm{KCl}$ concentration even up to $50 \mathrm{mM}$ (Figure $3 \mathrm{~A}$ ). $\mathrm{ZnCl}_{2}$ and $\mathrm{CdCl}_{2}$ induced significant loss of secondary structure of MtaIPMS. An exponential loss of CD ellipticity at $222 \mathrm{~nm}$ was observed with increasing concentration of $\mathrm{ZnCl}_{2}$ or $\mathrm{CdCl}_{2}$. However, a maximum loss of about 30 and 40 percent of CD signal was observed with about $2 \mathrm{mM} \mathrm{CdCl}_{2}$ and $\mathrm{ZnCl}_{2}$, respectively (Figure $3 \mathrm{~B}$ ). This suggests that interaction of $\mathrm{Cd}^{2+}$ and $\mathrm{Zn}^{2+}$ ions with MtaIPMS leads to only partial loss of secondary structure, i.e partial denaturation of the enzyme.

We wanted to understand the underlying mechanism for differential action of cations on both the structure and function of the MtaIPMS. The electrostatic surface potential of the enzyme as obtained from GRASP shows no large concentration of positive or negative charged regions on the enzyme surface (Figure 4). Hence, non-specific binding of cations on protein surface will be low. Furthermore, a differential effect of activation or inhibition and partial unfolding or no effect on the structure of enzyme by similarly charged cations like $\mathrm{Mg}^{2+}, \mathrm{Cd}^{2+}$ and $\mathrm{Zn}^{2+}$ was observed. These observations suggest a possibility of interaction of cations with similar charges at different sites in the enzyme. This possibility is further strengthened by the observation that $\mathrm{Cd}^{2+}$ and $\mathrm{Zn}^{2+}$ induce only partial unfolding but almost complete loss of functional activity 


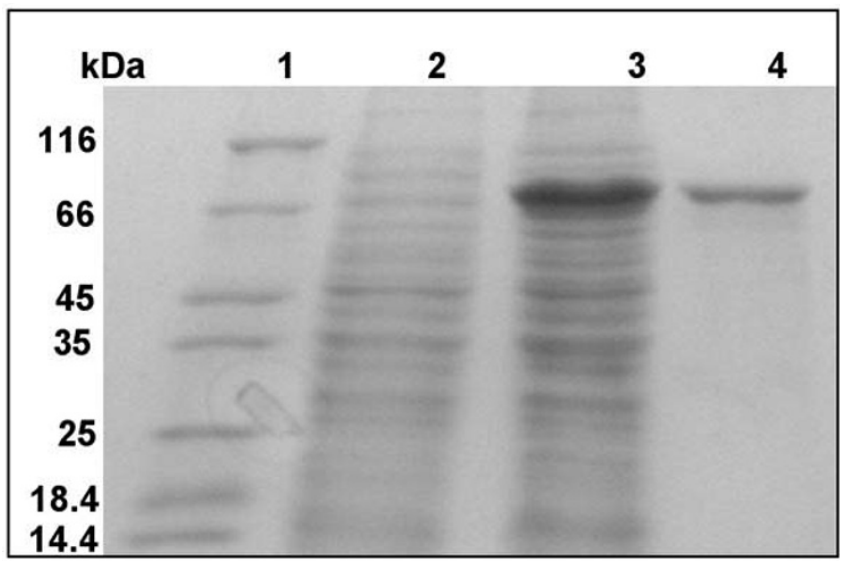

Figure I

Over-expression and purification of MtaIPMS. SDSPAGE analysis of cell lysate over-expressing MtaIPMS and purified protein. Lanes I-4 represent molecular weight markers, supernatant of un-induced cell lysate, supernatant of induced cell lysate and purified protein, respectively.

of the enzyme. So we concentrated our efforts on mapping site of interaction of various cations on MtaIPMS. To obtain an isolated folded catalytic domain of the enzyme, limited proteolysis technique was applied which has been demonstrated by us to be a technique of choice for obtaining isolated domain/s in folded functionally active form from the full-length protein [11-13].

\section{Identification of a structural domain of Mt $\alpha$ IPMS}

Figure 5 summarizes the SDS-PAGE profile of the protein fragments obtained on limited proteolysis of recombinant MtaIPMS with $\alpha$-chymotrypsin. A major band (Band I) along with three minor bands of lower molecular weight proteins/fragments were observed. The Band I purified by SEC $^{1}$ showed a molecular mass of $46.6 \mathrm{kDa}$ (as determined by ESI-MS). There are about 47 cleavage sites for $\alpha$-chymotrypsin spread all over the primary sequence of MtaIPMS however, on limited proteolysis, an intact domain of about $46 \mathrm{kDa}$ was obtained. This suggests that the $46 \mathrm{kDa}$ domain obtained on limited proteolysis of full length protein is in a folded conformation as several of the proteolytic sites present in it are buried in protein interior and not accessible to protease for cleavage under experimental conditions.

The Band I showed no affinity for the nickel-nitrilotriacetic acid-agarose matrix and anti-His antibody on western (Data not shown) suggesting that it corresponds to portion of MtaIPMS in which $\mathrm{N}$-terminus has been removed as the histidine tag is attached to the $\mathrm{N}$-terminus of the full-length protein. For determining the site of cleavage of protease in the protein, $\mathrm{N}$-terminal sequencing of the obtained fragment was carried out. It had a sequence
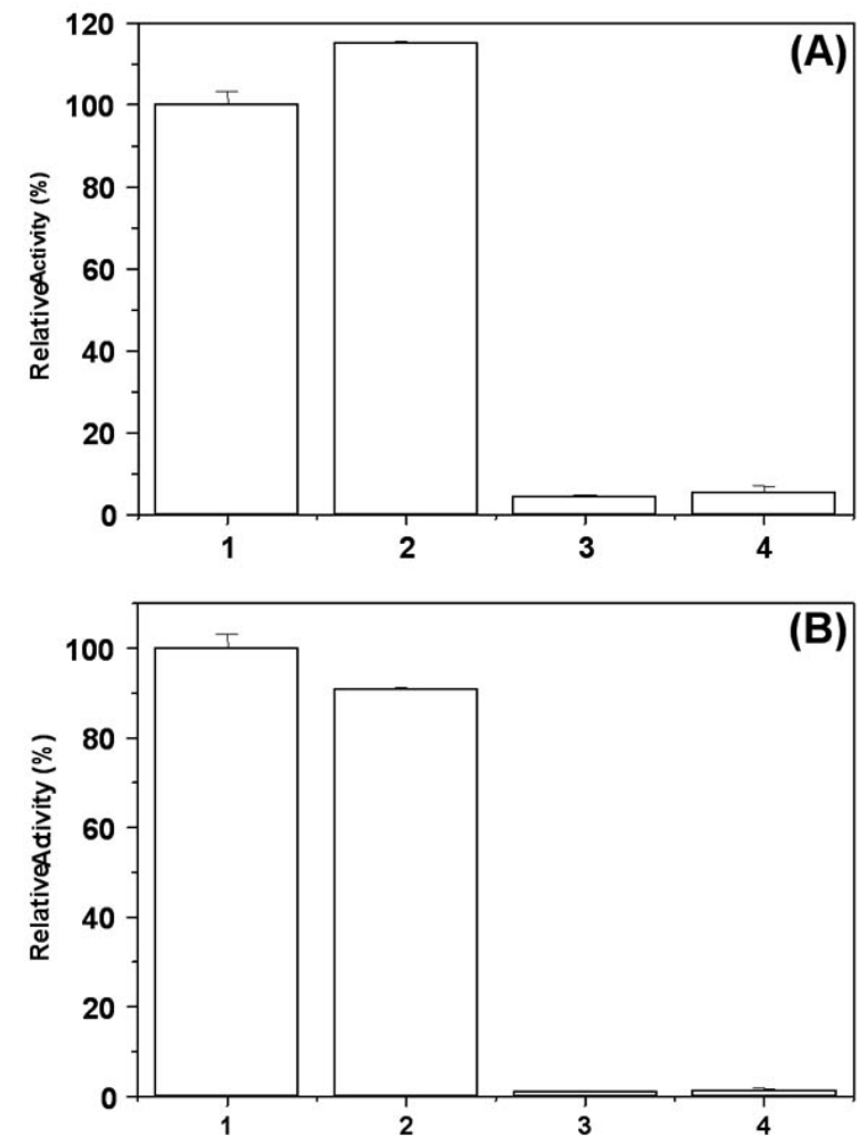

Figure 2

Cations influence enzymatic activity of both MtoIPMS and the TIM barrel domain. Effect of $\mathrm{KCl}$, $\mathrm{MgCl}_{2}, \mathrm{ZnCl}_{2}$ or $\mathrm{CdCl}_{2}$ on enzymatic activity of $\mathrm{Mt \alpha}$ IPMS (Panel A) and the catalytic TIM barrel Domain (Panel B). In both the panels the bars I to 4 represent samples of protein in presence of $20 \mathrm{mM} \mathrm{KCl}, 20 \mathrm{mM} \mathrm{KCl}+5 \mathrm{mM} \mathrm{MgCl}, 20$ $\mathrm{mM} \mathrm{KCl}+5 \mathrm{mM} \mathrm{MgCl} 2+2 \mathrm{mM} \mathrm{ZnCl}_{2}$ and $20 \mathrm{mM} \mathrm{KCl}+5$ $\mathrm{mM} \mathrm{MgCl}{ }_{2}+2 \mathrm{mMCdCl}$, respectively. The data is represented as percent activity with the activity of the protein in presence of $20 \mathrm{mM} \mathrm{KCl}$ taken as $100 \%$. The data is represented as mean $\pm S D$ of three values.

RPFAE. Based on N-terminal sequence and the observed molecular mass, Band I corresponds to the protein fragment Arg47 to Phe457 of the MtaIPMS. This fragment consists of the TIM barrel portion, sub domain I and a small part of sub domain II of the full-length enzyme (Figure 6). It has been designated as the TIM barrel domain in this study.

\section{The TIM barrel domain of Mt $\alpha$ IPMS is stabilized as a functionally active dimer}

To confirm that the isolated TIM barrel domain is stabilized in folded conformation, structural studies were carried out. Figure $7 \mathrm{~A}$ and $7 \mathrm{~B}$, shows far UV-CD and 

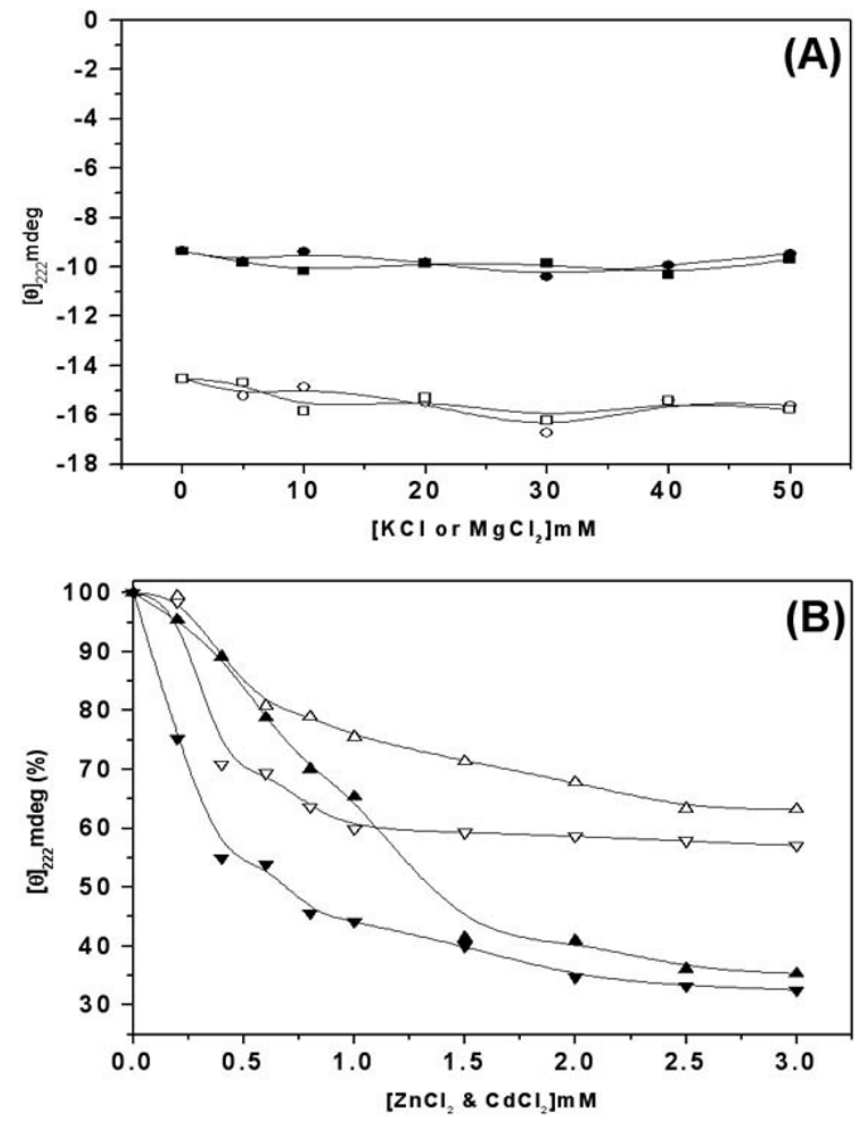

Figure 3

Divalent cations have differential effects on the secondary structure of Mt $\alpha$ IPMS and TIM barrel domain. A. Effect of increasing concentration of $\mathrm{KCl}$ (circle) and $\mathrm{MgCl}_{2}$ (square) on the CD ellipticity at $222 \mathrm{~nm}$ of MtaIPMS (open symbols) and TIM barrel domain (closed symbol). B. Effect of increasing concentration of $\mathrm{ZnCl}_{2}$ (up triangle) and $\mathrm{CdCl}_{2}$ (down triangle) on the $\mathrm{CD}$ ellipticity at $222 \mathrm{~nm}$ of MtalPMS (open symbols) and TIM barrel domain (closed symbols).

tryptophan fluorescence spectra respectively, of the TIM barrel domain. A far-UV profile typical of a $\alpha \beta$-protein [14] was observed. The tryptophan fluorescence emission spectra of the domain showed emission wavelength maxima at about $334 \mathrm{~nm}$ suggesting that the tryptophan moieties in the TIM barrel domain are buried in the hydrophobic core of the protein [15]. These observations support the suggestion that the isolated TIM barrel domain is stabilized in folded conformation under physiological conditions.

The most convincing result proving that the isolated TIM barrel domain is stabilized in folded conformation similar to that in which it is present in the full-length protein came from thermal denaturation studies. Figure $7 \mathrm{C}$ shows the comparative thermal denaturation profile of native
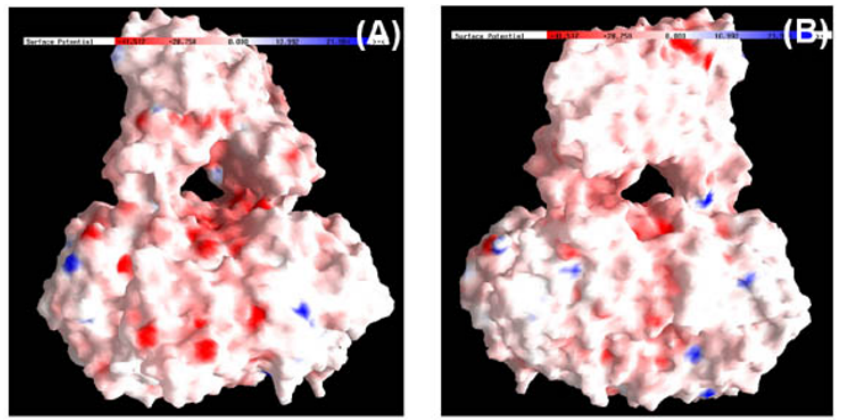

Figure 4

Electrostatic potential of molecular surface of MtaIPMS dimer. The colors blue and red represents negative and positive potential. Panels $A$ and $B$ represent MtalPMS dimer molecule in two different orientations. The molecular surface was displayed using GRASP.

Mt $\alpha$ IPMS and isolated TIM barrel domain as studied by monitoring the loss of CD signal at $222 \mathrm{~nm}$ at increasing temperature. For MtaIPMS at pH 7.5, a single sigmoidal transition centered at about $49^{\circ} \mathrm{C}$ and corresponding to loss of only about $35 \%$ of the CD signal was observed. For the TIM barrel domain, a biphasic transition with a main transition having Tm about $52^{\circ} \mathrm{C}$ corresponding to loss of about $50 \%$ structure of the protein along with a minor transition with $\mathrm{Tm}$ about $92^{\circ} \mathrm{C}$ and loss of about $70 \% \mathrm{CD}$ signal was observed. The similar thermal denaturation profile for the main transition of TIM barrel domain and the heat sensitive domain of the MtaIPMS indicates that they correspond to same portion of the full-length protein i.e. the catalytic N-terminal domain. This is confirmed by complete loss of functional activity of both the full-length enzyme and isolated TIM barrel domain at $65^{\circ} \mathrm{C}$ (Figure

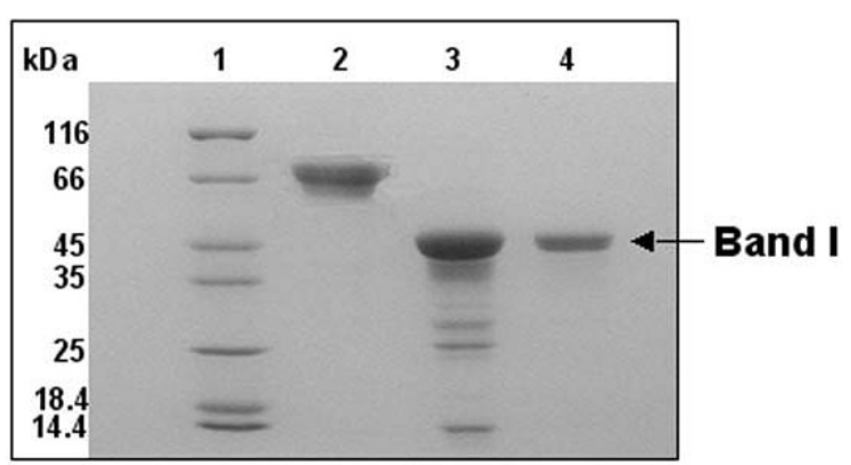

Figure 5

Limited proteolysis of Mt $\alpha$ IPMS with $\alpha$-chymotrypsin at $\mathbf{p H ~} 7.5$ and $25^{\circ} \mathrm{C}$. SDS-PAGE profile of the protein fragment(s) obtained on limited proteolysis of recombinant Mt $\alpha$ IPMS with $\alpha$-chymotrypsin. Lanes I-4 represent molecular weight markers, undigested MtalPMS, protein digested with $\alpha$-chymotrypsin and purified Band I, respectively. 


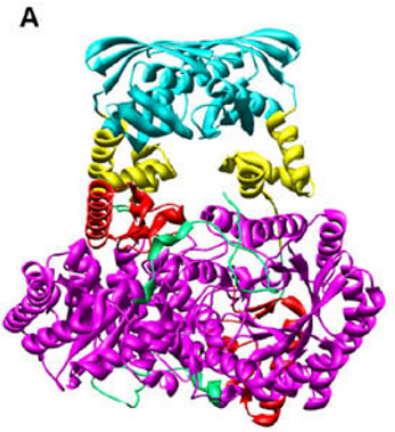

B

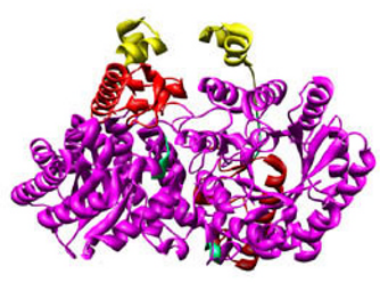

Figure 6

MOLSCRIPT model of Mt $\alpha$ IPMS (Panel A) and the TIM barrel domain (Panel B). The TIM barrel domain (magenta), sub-domain I (yellow), sub-domain II (red) and regulatory domain (cyan). The model has been generated using program UCSF Chimera.

7D) suggesting that it is indeed the catalytic domain that is unfolded under these conditions. These observations conclusively demonstrate that isolated catalytic TIM barrel domain is stabilized in a conformation similar to that in, which it is present in native conformation in the fulllength protein.

The quaternary structure of the isolated TIM barrel domain was obtained by SDS-PAGE analysis of glutaraldehyde crosslinked sample, Figure 7E. A molecular mass of about $93 \mathrm{kD}$ was observed for the cross-linked sample, which is about twice the mass of about $46.6 \mathrm{kDa}$ observed for this fragment. Hence, isolated TIM barrel domain is stabilized as a dimer under physiological conditions.

According to crystal structure, TIM barrel domain of MtaIPMS is the catalytic domain and it contains all the residues required for the catalytic activity of enzyme [4] so we carried out a functional activity assay on it. The TIM barrel domain did show functional activity however; it was only about $12 \%$ of that observed for the MtaIPMS (Figure 7D). Furthermore, unlike MtaIPMS whose functional activity was significantly inhibition by L-leucine, the functional activity of TIM barrel domain was not affected by L-leucine (Figure 7D, inset). X-ray studies have demonstrated that L-leucine binds to the regulatory domain of the enzyme [4][16,17] which is absent in the isolated TIM barrel domain, explaining why L-leucine did not inhibit the activity of the catalytic domain. These results demonstrate that the TIM barrel domain obtained on limited proteolysis of the MtaIPMS with $\alpha$-chymotrypsin is indeed the catalytic domain of the enzyme and it can carry out the catalytic function on its own.

\section{Differential effect of metal cations on the functional activity and structural properties of isolated TIM barrel domain of Mt $\alpha$ IPMS}

Figure 2B summarizes the effect of $\mathrm{KCl}, \mathrm{MgCl}_{2}, \mathrm{ZnCl}_{2}$ and $\mathrm{CdCl}_{2}$ on functional activity of TIM barrel domain. Monovalent cation $\mathrm{K}^{+}$, was found to be essential for functional activity as in its absence no activity was observed (Figure 2A). The $\mathrm{Mg}^{2+}$ ions did not show any further enhancement in activity observed in presence of $\mathrm{K}^{+}$ions. The $\mathrm{Zn}^{2+}$ and $\mathrm{Cd}^{2+}$ ions showed inhibition of functional activity of the isolated TIM barrel domain with almost complete loss observed at about $3 \mathrm{mM} \mathrm{ZnCl}{ }_{2}$ or $\mathrm{CdCl}_{2}$.

Figure 3 shows the changes in the far-UV CD of the TIM barrel domain at $222 \mathrm{~nm}$ in presence of increasing concentration of $\mathrm{MgCl}_{2}, \mathrm{KCl}, \mathrm{ZnCl}_{2}$ or $\mathrm{CdCl}_{2}$. The addition of $\mathrm{MgCl}_{2}$ or $\mathrm{KCl}$, even up to $50 \mathrm{mM}$ concentration, gave no significant alteration in the secondary structure of the protein (Figure 3B). However, with increasing concentration of $\mathrm{ZnCl}_{2}$ or $\mathrm{CdCl}_{2}$ an exponential loss of CD signal at 222 nm was observed and at about $2 \mathrm{mM} \mathrm{ZnCl}_{2}$ or $\mathrm{CdCl}_{2}$, about 70 percent loss of secondary structure of the TIM barrel domain was observed.

\section{Divalent cations induce structural cooperativity in the Mt $\alpha$ IPMS}

To analyze whether the two structural domains, N-terminal or TIM barrel domain and the C-terminal or regulatory domain, of MtaIPMS interact strongly with each other or they are independent folding/unfolding units of the protein, thermal denaturation studies were carried out by monitoring the loss of secondary structure of enzyme at increasing temperatures. Figure 8 shows the changes in CD ellipticity at $222 \mathrm{~nm}$ for MtaIPMS as a function of temperature. A broad sigmoidal transition with an apparent Tm of about $49^{\circ} \mathrm{C}$ was observed. An interesting observation was that a loss of only about 35\% CD ellipticity at $222 \mathrm{~nm}$ was associated with the thermal denaturation of MtaIPMS, demonstrating that the major part of the protein molecule is resistant to thermal unfolding. This indicates that MtaIPMS is composed of two unfolding units that behave independently and have different thermal stabilities: one being sensitive to thermal denaturation and the other resistant to it. A similar sensitivity of structural domains to thermal denaturation has been observed for other proteins [13]. Hence, full-length Mt $\alpha$ IPMS is structurally a non-cooperative molecule having two distinct structural units that fold/unfold independently of each other.

The effect of cations on the thermal denaturation of Mt $\alpha$ IPMS was studied by incubating the protein with salts like $\mathrm{KCl}, \mathrm{MgCl}_{2}, \mathrm{CdCl}_{2}$ or $\mathrm{ZnCl}_{2}$. As all these salts contain the same anion (i.e. $\mathrm{Cl}^{-}$), the different effects observed in the comparative study using these salts will be mainly due 

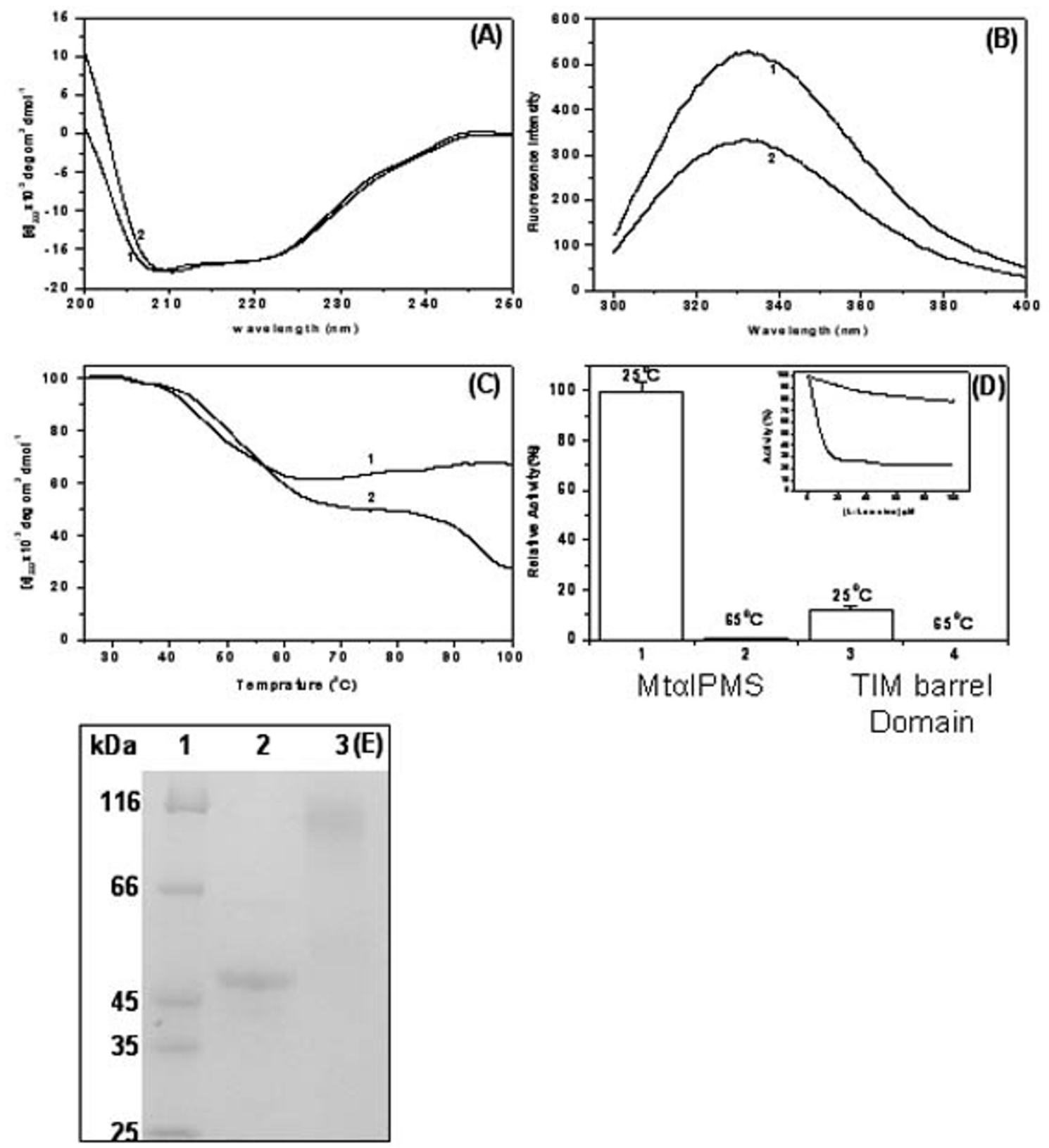

Domain

\section{Figure 7}

Structural and functional properties of Mt $\alpha$ IPMS and the TIM barrel domain. A. Far-UV CD spectra of MtalPMS (profile I) and the TIM barrel domain (profile 2). B. Tryptophan emission spectra of MtaIPMS (profile I) and the TIM barrel domain (profile 2). C. Thermal unfolding of MtalPMS and the TIM barrel domain. Effect of increasing temperature on the CD ellipticity at $222 \mathrm{~nm}$ of MtaIPMS (profile I) and the TIM barrel domain (profile 2), respectively. The values have been represented as percentage with the value obtained at $25^{\circ} \mathrm{C}$ for each sample taken as 100 percent, respectively. $\mathbf{D}$. Enzymatic activity of MtoIPMS and the TIM barrel domain. Relative enzymatic activity of MtalPMS (Bar I and 2) and the TIM barrel domain (Bar 3 and 4) at 25 and $65^{\circ} \mathrm{C}$, respectively. The values have been represented as percent activity with the value observed for MtalPMS at $25^{\circ} \mathrm{C}$ taken as $100 \%$. The data is represented as mean \pm SD of three values. Inset shows the loss of enzymatic activity of the MtaIPMS (closed symbols) and the TIM barrel domain (open symbols) on incubation with increasing concentration of L-lecucine. The data has been represented as percentage with the activity of Mt $\alpha$ IPMS or the TIM barrel domain in absence of Leucine taken as 100 percent. E. SDS-PAGE profile of glutaraldehyde cross-linked TIM barrel domain. Lanes I-3 represent molecular weight markers, uncrosslinked and glutaraldehyde cross-linked TIM barrel domain, respectively. 
to different cations. Figure 8 summarizes the thermal denaturation profiles of $\mathrm{KCl}-, \mathrm{MgCl}_{2^{-}}, \mathrm{CdCl}_{2^{-}}, \mathrm{ZnCl}_{2^{-}}$or

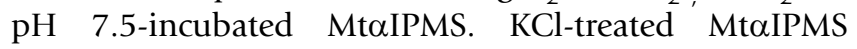
showed a thermal denaturation profile similar to that observed for the enzyme in absence of salt demonstrating that in presence of $\mathrm{KCl}$ the enzyme still behaves noncooperatively. However, for $\mathrm{MgCl}_{2^{-}}, \mathrm{CdCl}_{2^{-}}$or $\mathrm{ZnCl}_{2^{-}}$ treated Mt $\alpha$ IPMS, a single transition with almost complete loss of secondary structure was observed. This demonstrates that divalent cations induce strong interactions between the structural domains of enzyme resulting in induction of cooperativity in the otherwise non-cooperative MtaIPMS molecule. An interesting observation was that the Tm associated with the $\mathrm{CdCl}_{2}$-treated Mt $\alpha$ IPMS (about $49^{\circ} \mathrm{C}$ ) is significantly lower than that of $65^{\circ} \mathrm{C}$ observed for $\mathrm{MgCl}_{2}$-treated MtaIPMS. These differences in $\mathrm{Tm}$ in the presence of different salts are due to the differential effect of salts on the enzyme structure. The $\mathrm{CdCl}_{2}$ induces partial denaturation of the enzyme whereas, the $\mathrm{MgCl}_{2}$ does not affect the structure of enzyme significantly. The information that divalent cations induce cooperativity in the otherwise non-cooperative MtaIPMS molecule is of importance as it suggests that $\mathrm{Mg}^{2+}$ ions that are included in the enzyme assay induce interaction between the catalytic $\mathrm{N}$-terminal and the regulatory C-terminal domain as a result of which the regulatory domain can modulate the activity of the catalytic domain.

\section{Discussion}

MtaIPMS in the functional form, is an intimately associated extended dimer [4]. The dimer interface of the enzyme covers only the $\mathrm{N}$ - and C-terminal domains which are in contact with each other in the native conformation of the enzyme [4]. The two TIM barrels in the dimer of enzyme pack against one another and are tied together by $\mathrm{N}$-and $\mathrm{C}$-terminal extensions. The $\mathrm{N}$-terminal residues $18-50$, from one monomer wind over the surface of the other monomer. The C-terminal domain of the enzyme also dimerizes. The TIM barrel without the $\mathrm{N}$ and the C-terminal extension contribute only $12 \%$ of the total buried surface as compared to $80 \%$ of the total buried surface area attributed by the dimerization of the barrel including the $\mathrm{N}$ - and C-terminal extensions [4]. It has been demonstrated in this paper that the isolated TIM barrel domain (Arg47 to Phe457) that is devoid of the N-terminal extension and the C-terminal domain, is stabilized as a functionally active dimer. This suggests that the $\mathrm{N}$-terminal extension and the regulatory C-terminal domain are not essential for the stabilization of the dimeric configuration of Mt $\alpha$ IPMS under physiological conditions, which has also been indicated earlier [4].

The functional activity of the MtaIPMS is subject to major controls by small molecules. Metal cations, $\mathrm{K}^{+}$and $\mathrm{Mg}^{2+}$ are the activators of Mt $\alpha$ IPMS and their presence is impor-

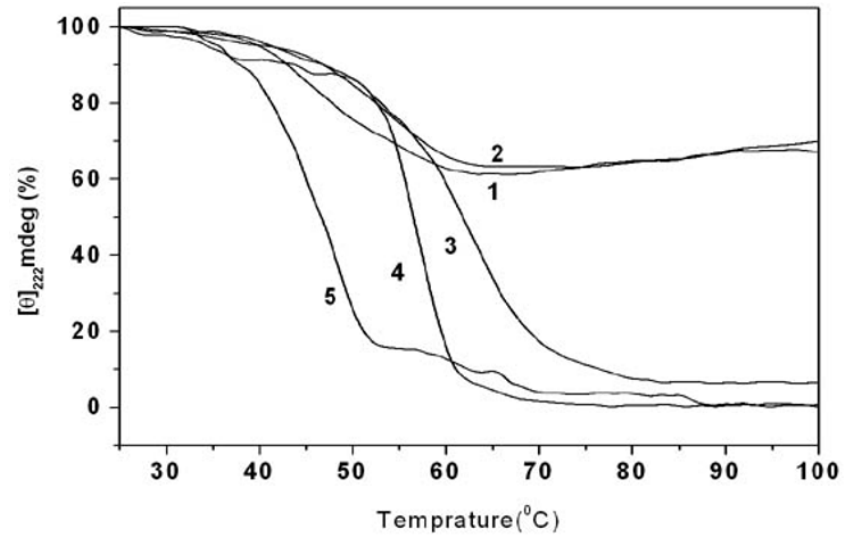

\section{Figure 8}

Thermal unfolding of salt-treated Mt $\alpha$ IPMS. Effect of increasing temperature on the CD ellipticity at $222 \mathrm{~nm}$ of MtoIPMS (profile I), MtaIMPS in presence of $5 \mathrm{mM} \mathrm{KCl}$ (profile 2), $5 \mathrm{mM} \mathrm{MgCl}_{2}$ (profile 3), $0.25 \mathrm{mM} \mathrm{ZnCl}_{2}$ (profile 4) and $0.25 \mathrm{mM} \mathrm{CdCl}_{2}$ (profile 5), respectively. The values have been represented as percentage with the value obtained at $25^{\circ} \mathrm{C}$ for each sample taken as 100 percent, respectively.

tant for the activity of the enzyme [9]. The activating molecules influence the oligomeric structure or conformation of the enzyme belonging to the family of Claisen-condensing enzymes, including several $\alpha$ IPMS [18-20]. However, our studies and a recent report [10] demonstrates that for Mt $\alpha$ IPMS, both the activators $\mathrm{K}^{+}$and $\mathrm{Mg}^{2+}$, have no significant effect on the oligomeric or the secondary structure of the enzyme. In contrast, $\mathrm{Zn}^{2+}$ and $\mathrm{Cd}^{2+}$ induced partial and almost complete denaturation of the MtaIPMS and the catalytic TIM barrel domain, respectively. However, complete loss of enzymatic activity was observed for both the full-length enzyme and isolated catalytic domain under these conditions. These observations suggest that $\mathrm{Zn}^{2+}$ and $\mathrm{Cd}^{2+}$ bind directly to the catalytic domain of the enzyme leading to its unfolding/denaturation resulting in loss of activity. Binding of $\mathrm{Zn}^{2+}$ ions leading to unfolding of the protein has recently been reported for spermadhesion PSP-I [21]. The activator $\mathrm{K}^{+}$ions induces activation of both the full-length enzyme as well as the isolated catalytic TIM barrel domain suggesting that it interacts directly with the catalytic domain and induce activation of the enzyme. These results for the first time demonstrate that different cations bind at different sites in the Mt $\alpha$ IPMS resulting in differential modulation of functional and/or structural property of the enzyme.

One important observation was that the isolated TIM barrel domain showed only $12 \%$ activity of that observed for the MtaIPMS although the structural and stability studies showed that the isolated domain has similar conforma- 
tion as that in which it is present in the native MtaIPMS. This suggests the possibility that probably the C-terminal domain of enzyme is necessary for optimum activity of the enzyme. In other words the non-catalytic C-terminal domain modulates the activity of the catalytic TIM barrel domain and its absence results in decrease in activity of the catalytic domain. This possibility is supported by the fact that L-leucine, which binds in the C-terminal domain, inhibits the activity of the MtaIPMS.

An important property of MtaIPMS found to be influenced by the cations is the structural cooperativity of the enzyme molecule. Divalent cations were found to induce cooperativity in the otherwise structurally non-cooperative Mt $\alpha$ IPMS molecule. The induction of cooperativity in the Mt $\alpha$ IPMS molecule by the $\mathrm{Mg}^{2+}$, which is an activator of the enzyme and is added to the reaction mixture during activity measurements, may help in modulation of functional activity of the TIM barrel domain by the regulatory domain.

\section{Conclusion}

Comparative studies on the effect of cations on the structural and functional properties of MtaIPMS and its isolated catalytic TIM barrel domain demonstrates that different cations interact at different sites in the enzyme molecule inducing different effects on the enzyme structure. $\mathrm{Zn}^{2+}$ and $\mathrm{Cd}^{2+}$ ions interact with the catalytic domain of the enzyme and induce unfolding/denaturation of the domain and loss of its functional activity. The physiological activator $\mathrm{K}^{+}$ion also interacts with the catalytic domain and activates the enzyme however; it does not induce any significant structural change in the enzyme. The divalent cations were found to induce structural cooperativity in the otherwise non-cooperative Mt $\alpha$ IPMS molecule. The study provides an intriguing insight into the structural details of the enzyme MtaIPMS and its regulation by the cations. This information will be of significant importance in designing of the inhibitors of the enzyme.

\section{Methods \\ Materials}

All chemicals used in the study were purchased from Sigma-Aldrich Chemical Company St. Louis, USA, and were of highest purity available. All chromatographic columns were purchased from GE Healthcare Biosciences, with the exception of Ni-NTA agarose and chelex-100 that were purchased from Qiagen and BioRad, respectively.

\section{Overproduction and purification of Mt $\alpha$ IPMS}

The overproduction and purification of recombinant Mt $\alpha$ IPMS was carried out with slight modification of the earlier described method [22]. Mt $\alpha$ IPMS was overproduced in E. coli $\mathrm{C} 41$ harboring the plasmid pProEX-HTa. Cells were grown in 2XYT medium with $100 \mathrm{mg} /$ litre ampicillin and induced in mid log phase with $0.5 \mathrm{mM}$ IPTG for 15 hours at $16^{\circ} \mathrm{C}$ for the synthesis of Mt $\alpha$ IPMS. The overexpressed protein was purified by earlier reported procedure [22]. The purified protein showed $>95 \%$ purity, as observed by ESI-MS and SDS-PAGE analysis, and was stored in $50 \mathrm{mM}$ HEPES pH 7.5. Purified protein and all the buffers used in the study were subjected to divalent metal removal using chelex-100 resin.

\section{Assay for enzymatic activity}

The enzymatic activity of MtaIPMS and TIM barrel domain were determined using 4,4'-dithiodipyridine (DTP) to detect the formation of coenzyme A (CoA) at $324 \mathrm{~nm}\left(\varepsilon=19800 \mathrm{M}^{-1} \mathrm{~cm}^{-1}\right)$ at $25^{\circ} \mathrm{C}$ as reported earlier [23]. A typical reaction mixture contained $50 \mathrm{mM}$ HEPES (pH 7.5), $20 \mathrm{mM} \mathrm{KCl,} 5$ mM MgCl $2,100 \mu \mathrm{M}$ DTP, $300 \mu \mathrm{M}$ AcCoA, $300 \mu \mathrm{M} \alpha$-KIV and $20 \mathrm{nM}$ of Mt $\alpha$ IPMS or TIM barrel domain. Reaction mixtures were incubated for $4 \mathrm{~h}$ at $25^{\circ} \mathrm{C}$ with salts and reactions were initiated by addition of $\alpha$-KIV. For studies with isolated TIM barrel domain higher concentrations of AcCoA and $\alpha$-KIV was also tried but no significant change in the measured enzymatic activity was observed.

To probe the effect of thermal changes on the full-length enzyme and the isolated TIM barrel domain, samples were heated for $15 \mathrm{~min}$ at the desired temperature before measurements were made.

\section{ESI-MS}

The mass spectra were recorded on a MICRO-MASS QUATTRO II mass spectrometer (Micromass, Altricem, United Kingdom) equipped with an electrospray ionization (ESI) ion source as describe earlier [24].

\section{Limited proteolysis and purification of TIM barrel domain} $2.0 \mathrm{mg} / \mathrm{ml}$ protein was subjected to limited proteolysis with $\alpha$-chymotrypsin, at a protease to protein ratio of 1:50 $(\mathrm{w} / \mathrm{w})$ for $1 \mathrm{~h}$ at $25^{\circ} \mathrm{C}$. The reaction was stopped by addition of phenylmethylsulfonyl fluoride to a final concentration of $1 \mathrm{mM}$, and the samples were analyzed on $10 \%$ SDS-PAGE. For purification of TIM barrel domain, proteolysed samples were loaded on the Superdex 200 HR 10/ 300 column interfaced with AKTA FPLC (GE Healthcare Biosciences). The column was pre-equilibrated and run with $50 \mathrm{mM}$ HEPES, $\mathrm{pH}$ 7.5. $500 \mu \mathrm{l}$ of the sample was injected in the column and run at $25^{\circ} \mathrm{C}$ at a flow rate of $0.3 \mathrm{ml} / \mathrm{min}$, with detection at $280 \mathrm{~nm}$.

\section{Fluorescence spectroscopy}

Fluorescence spectra were recorded with Perkin-Elmer LS 50B spectroluminescencemeter in a $5 \mathrm{~mm}$ path length quartz cell. Protein concentration was $0.75 \mu \mathrm{M}$ for all experiments and the measurements were carried out at $25^{\circ} \mathrm{C}$. For monitoring tryptophan fluorescence the excita- 
tion wavelength of $280 \mathrm{~nm}$ was used and the spectra were recorded between 300 to $400 \mathrm{~nm}$.

\section{Circular dichroism measurements}

CD measurements were made with a Jasco J810 spectropolarimeter calibrated with ammonium (+)-10-camphorsulfonate. The results are expressed as the mean residual ellipticity $(\theta)$, which is defined as $(\theta)=100 \times$ $\theta_{\text {obs }} /(\mathrm{lc})$, where $\theta_{\text {obs }}$ is the observed ellipticity in degrees, $c$ is the concentration in mol residue per litre, and $l$ is the length of the light path in centimeters. The CD spectra were measured at an enzyme concentration of $0.75 \mu \mathrm{M}$ with a $1 \mathrm{~mm}$ cell at $25^{\circ} \mathrm{C}$. The values obtained were normalized by subtracting the baseline recorded for the buffer having the same concentration of salt under similar conditions.

\section{Metal ion induced structural alterations}

For studying the cation induced structural alterations, protein $(0.75 \mu \mathrm{M})$, except in case of $\mathrm{ZnCl}_{2}$ and $\mathrm{CdCl}_{2}(0.5 \mu \mathrm{M}$ of protein), in $5 \mathrm{mM}$ HEPES ( $\mathrm{pH} 7.5$ ) was incubated in the presence/absence of increasing concentration of $\mathrm{KCl}$, $\mathrm{MgCl}_{2}, \mathrm{ZnCl}_{2}$ and $\mathrm{CdCl}_{2}$ for $4 \mathrm{~h}$ at $4^{\circ} \mathrm{C}$ before the measurements were made. The $\mathrm{pH}$ of the solution was maintained throughout the studies.

\section{Thermal denaturation}

Thermal denaturation of Mt $\alpha$ IPMS $(0.75 \mu \mathrm{M})$ was monitored by change in molar ellipticity at $222 \mathrm{~nm}$ as function of temperature on a Jasco J810 spectropolarimeter equipped with peltier temperature controller system. The measurements were carried out in $5 \mathrm{mM}$ HEPES buffer $\mathrm{pH}$ 7.4. Samples were heated at constant rate of $1^{\circ} \mathrm{C} / \mathrm{min}$ in 1 mm cell.

\section{Cross linking using glutaraldehyde}

The TIM barrel domain $(0.2 \mathrm{mg} / \mathrm{ml})$ was incubated with $1 \%$ glutaraldehyde (final concentration) for $20 \mathrm{~min}$ at $4{ }^{\circ} \mathrm{C}$ followed by quenching of crosslinking reaction by addition of $2 \mu \mathrm{l}$ of $\beta$-mercaptoethanol. The cross-linked products were concentrated by centricon $(50 \mathrm{kDa}$, Millipore) and run on $8 \%$ SDS-PAGE for analysis.

\section{Abbreviations}

$\alpha$-IPMS, $\alpha$-isopropylmalate synthase, $\alpha$-KIV, $\alpha$-ketoisovalerate, SEC, Size exclusion chromatography, ESI-MS, Electrospray ionization mass spectrometry, AcCoA, Acetyl-Co A.

\section{Authors' contributions}

KS carried out all the experiments and the data analysis. VB conceived the study, participated in the design of the experiments and drafted the manuscript. Both the authors approved the final manuscript.

\section{Additional material}

\section{Additional File 1}

Effect of $\mathrm{ZnCl}_{2}$ and $\mathrm{CdCl}_{2}$ on secondary structure of MtoIPMS and TIM barrel domain. Far-UV CD spectra of MtoIPMS (Panel A and B) and TIM barrel domain (Panel $C$ and $D$ ) in presence of increasing concentration of $\mathrm{ZnCl}_{2}$ (Panel $\mathrm{A}$ and $\mathrm{C}$ ) and $\mathrm{CdCl}_{2}$ (Panel B and D). The various curves represent $\mathrm{ZnCl}_{2}$ and $\mathrm{CdCl}_{2}$ concentration of $0 \mathrm{mM}$ (solid line), $0.5 \mathrm{mM}$ (dashed line), $1 \mathrm{mM}$ (dotted line), $2 \mathrm{mM}$ (dashed dotted line), and $3 \mathrm{mM}$ (small dashed line).

Click here for file

[http://www.biomedcentral.com/content/supplementary/14726807-7-39-S1.jpeg]

\section{Acknowledgements}

We thank to Dr. C.M. Gupta for providing constant support during the studies. We are grateful to Dr. E.N. Baker for providing the clone for MtaIPMS used in the study. KS thanks CSIR, New Delhi, for financial assistance.

\section{References}

I. Kochi A: The global tuberculosis situation and the new control strategy of the World Health Organization. Bull World Health Organ 200I, 79:7I-75.

2. Rattan A, Kalia A, Ahmad N: Multidrug-resistant Mycobacterium tuberculosis: molecular perspectives. Emerg Infect Dis 1998, 4:195-209.

3. Hondalus MK, Bardarov S, Russel R, Chan J, Jacobs WRJr, Bloom BR: Attenuation of and protection induced by a leucine auxotroph of Mycobacterium tuberculosis. Infect Immun 2000, 68:2888-2898.

4. Koon N, Squire CJ, Baker EN: Crystal structure of LeuA from Mycobacterium tuberculosis, a key enzyme in leucine biosynthesis. Proc Nat Acad Sci USA 2004, I $01: 8295-8300$.

5. Kohlhaw G, Leary TR, Umbarger HE: Alpha-isopropylmalate synthase from Salmonella typhimurium. Purification and properties. J Biol Chem 1969, 244:22 I 8-2225.

6. Ulm EH, Bohme R, Kohlhaw G: Alpha-isopropylmalate synthase from yeast: purification, kinetic studies, and effect of ligands on stability. J Bacteriol I972, I I 0: I I I8-I I 26.

7. Chanchaem W, Palittapongarnpim P: A variable number of tandem repeats result in polymorphic alpha-isopropylmalate synthase in Mycobacterium tuberculosis. Tuberculosis (Edinb) 2002, 82: I-6.

8. Webster R, Gross S: The $\alpha$-Isopropylmalate Synthetase of Neurospora. I. The Kinetics and End Product Control of $\alpha$ Isopropylmalate Synthetase Function. Biochemistry 1965, 4:2309-23I8

9. de Carvalho LP, Blanchard JS: Kinetic analysis of the effects of monovalent cations and divalent metals on the activity of Mycobacterium tuberculosis alpha-isopropylmalate synthase. Arch Biochem Biophys 2006, 451 : I4I-I 48.

10. de Carvalho LP, Blanchard JS: Kinetic and chemical mechanism of alpha-isopropylmalate synthase from Mycobacterium tuberculosis. Biochemistry 2006, 45:8988-8999.

II. Akhtar MS, Krishnan MY, Bhakuni V: Insight into the mechanism of action of hyaluronate lyase: role of $\mathrm{C}$-terminal domain and $\mathrm{Ca}^{++}$in the functional regulation of enzyme. J Biol Chem 2006, 28I:28336-28344.

12. Mishra P, Akhtar MS, Bhakuni V: Unusual structural features of bacteriophge-associated hyaluronate lyase. J Biol Chem 2006, 281:7|43-7|50.

13. Bhatt AN, Shukla N, Aliverti A, Zanetti G, Bhakuni V: Modulation of cooperativity in Mycobacterium tuberculosis NADPHFerredoxin reductase: cation and $\mathrm{pH}$ induced alterations in native conformation and destabilization of the NADP+ binding domain. Protein Sci 2005, 14:980-992. 
14. Chen YH, Yang JTG, Martinez HM: Determination of the secondary structures of proteins by circular dichroism and optical rotatory dispersion. Biochemistry 1972, I I:4|20-4I3I.

15. Lackowicz JR: Principles of Fluorescence Spectroscopy New York: Plenum Press; 1983.

16. Kohlhaw GB: Alpha-isopropylmalate synthase from yeast. Methods Enzymol 1988, 166:4|4-423.

17. Wiegel J, Schlegel HG: Alpha-isopropylmalate synthase from Alcaligenes eutrophus H 16. III. Endproduct inhibition and its relief by valine and isoleucine. Arch Microbiol 1977, I | 4:203-210.

18. Tong EK, Duckworth HW: The quaternary structure of citrate synthase from Escherichia coli KI2. Biochemistry 1975, I4:235-24I.

19. Anstrom DM, Kallio K, Remington SJ: Structure of the Escherichia coli malate synthase G:pyruvate:acetyl-coenzyme A abortive ternary complex at I.95 A resolution. Protein Sci 2003, I 2: 1822-1832.

20. Smith CV, Huang CC, Miczak A, Russell DG, Sacchettini JC, zu Bentrup $\mathrm{KH}$ : Biochemical and structural studies of malate synthase from Mycobacterium tuberculosis. J Biol Chem 2003, 278: $1735-1743$.

21. Campanero-Rhodes M, Menendez M, Saiz JL, Sanz L, Calvet IJ, Solis D: Zinc ions induce the unfolding and self-association of boar spermadhesin PSP-I, a protein with a single CUB domain architecture, and promote its binding to heparin. Biochemistry 2006, 45:8227-8235.

22. Koon N, Squire CJ, Baker EN: Crystallization and preliminary $\mathbf{X}$ ray analysis of alpha-isopropylmalate synthase from Mycobacterium tuberculosis. Acta Cryst 2004, D60: 1767-1969.

23. de Carvalho LP, Argyrou A, Blanchard JS: Slow-onset feedback inhibition: inhibition of Mycobacterium tuberculosis alphaisopropylmalate synthase by L-leucine. J Am Chem Soc 2005, 1 27:10004-10005.

24. Ali V, Prakash K, Kulkarni S, Ahmad A, Madhusudan KP, Bhakuni V: 8anilino-I-naphthalene sulfonic acid (ANS) induces folding of acid unfolded cytochrome c to molten globule state as a result of electrostatic interactions. Biochemistry 1999, I I:4|20-4|3|.

Publish with Bio Med Central and every scientist can read your work free of charge

"BioMed Central will be the most significant development for disseminating the results of biomedical research in our lifetime. "

Sir Paul Nurse, Cancer Research UK

Your research papers will be:

- available free of charge to the entire biomedical community

- peer reviewed and published immediately upon acceptance

- cited in PubMed and archived on PubMed Central

- yours - you keep the copyright 